F. PHYSICAL PROPERTIES OF LUNAR SAMPLES 


\title{
INTERFEROMETRIC STUDIES ON APOLLO 11 AND APOLLO 12 LUNAR GLASS OBJECTS
}

\author{
S. TOLANSKY \\ Department of Physics, Royal Holloway College, Egham, Surrey, England
}

\begin{abstract}
A review of results obtained by an examination with optical interferometry of a variety of glassy objects found in lunar fines from Apollo 11 and 12. Glassy spheres, cylinders, chips and fragments have been examined. It is established that many spherules show

(a) evidence of shock;

(b) evidence of impact with a small particle;

(c) very high specularity.

Some small pieces of lunar glass reveal the characteristic step pattern shown by glass shattered in the laboratory.
\end{abstract}

\section{General Introduction}

This report is devoted to $5 \mathrm{gm}$ of Lunar fines from the Apollo 11 Expedition and three $0.5 \mathrm{gm}$ samples of fines from Apollo 12. The $5 \mathrm{gm}$ Apollo 11 sample will be discussed first. As received the fines had been pre-sieved through a $1 \mathrm{~mm}$ mesh sieve. By means of tweezers 207 glassy spherules and round-ended glassy cylinders were extracted. The majority of these objects (size range $1 \mathrm{~mm}$ to $0.1 \mathrm{~mm}$ ) were reddish-brown glass and many exhibited a very high specularity. Some of the larger objects were of a greyish metallic lustre. A few were highly coloured, some green, one a deep blue. Although a considerable proportion of the fines consists of fragmental glass, the extracted objects did not exceed in amount some $0.01 \%$ of the total. If this fines sample is representative, there exist more than 40000 such objects per $\mathrm{kg}$ of dust. This figure refers only to objects big enough to handle and extract. When smears of the fines are examined with higher microscope powers $(\times 500$ or more) then larger numbers of such objects appear, even up to 100 per $\mathrm{mg}$, i.e. the formidable number of $10^{8}$ per kg of Apollo 11 Lunar dust. Even this is well exceeded in the Apollo 12 samples (see later).

These specular objects and numerous small irregular glassy fragments also found in the dust have been examined at magnifications mostly up to $\times 1000$ and more, using $3 \mathrm{~mm}$ and $4 \mathrm{~mm}$ microscope objectives, with a specially designed interference microscope, whereby the chosen object is mounted on an inverted microscope and is matched against an interferometrically selected very thin glass cover slip. Some of the denser objects were matched against titanium-oxide coated glass cover slips to enhance interference fringe contrast. By using, variously, mercury green light, an unfiltered mercury light and white light, the object can be seen covered with interference fringes. Such fringes are in effect a micro-contour map of the surface wherein from fringe to fringe the height has changed by half a light wave ( $2730 \AA$ for green mercury). Using simple two-beam systems one has no difficulty in recognising height changes down to as small, perhaps, as $100 \AA$ or so. (With multiple-beam interference 
systems, one can resolve $5 \AA$ features, but there is as yet no justification for pursuing interferometry to such fine limits with these particular Lunar objects.)

Characteristic features of typical objects are worthy of consideration. Figure la shows typical spherules and Figure $1 \mathrm{~b}$ shows typical cylinders. (In each figure the largest object is $\frac{3}{4} \mathrm{~mm}$.) Many spherules have impacted crusty tails (Figure 1c) which suggest that the particle has struck the Lunar dust at high velocity. An appreciable fraction show micro impact craters (Figure 1d) which indicate having suffered collision with a small solid object. Tail formation and crater production have clearly different origins. If it be postulated that many of the spherules have been high-speed

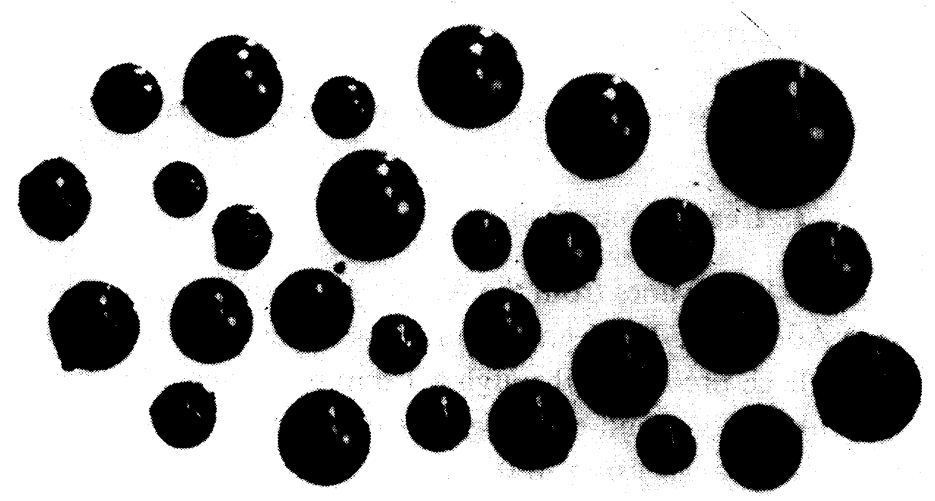

1a.

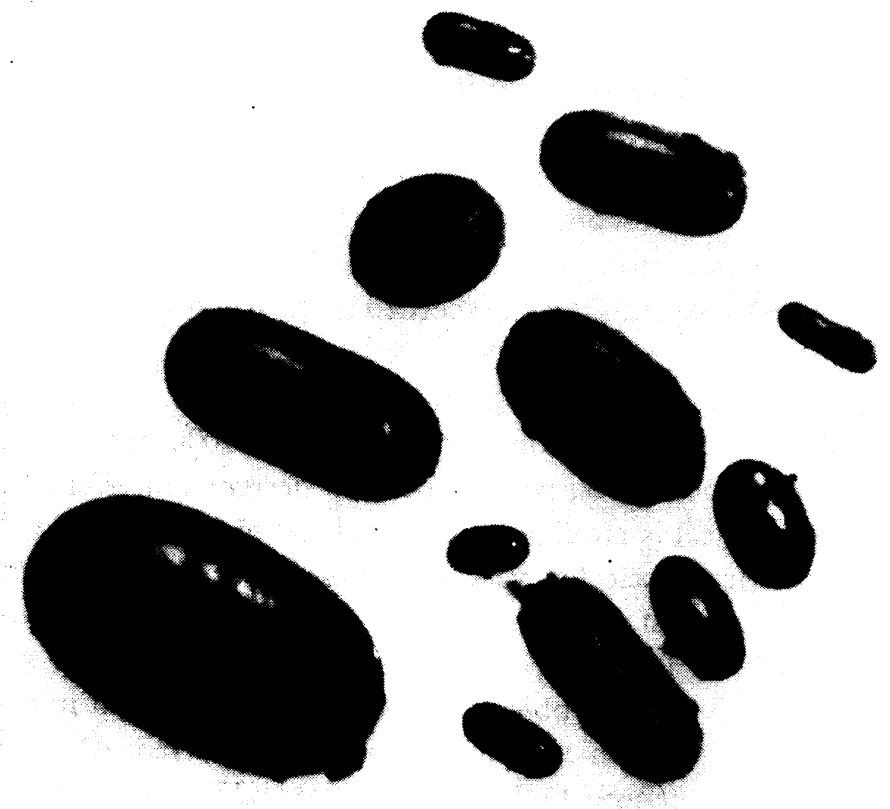

lb.

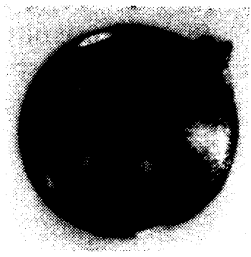

lc.

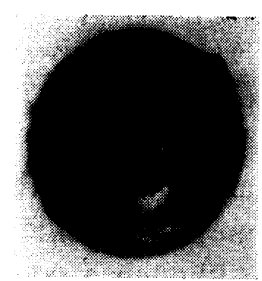

ld.

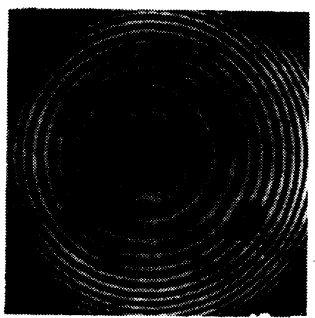

le.

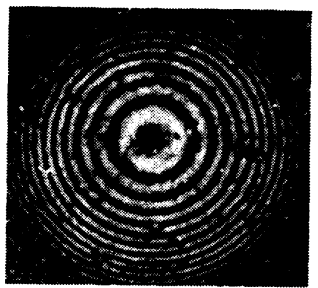

If.

Fig. 1a-f. 
projectiles, the tails can originate as a sintered mass formed by landing on dust. The craters suggest that such spherules have been hurled through a cloud of small solid particles.

\section{Interferograms. Apollo 11, Sample No. 10084.15}

\section{A. SPHERULES}

There are optical difficulties associated with photographing interference fringe systems from spheres of diameter $0.1 \mathrm{~mm}$ and less, due to small working distances of lenses, but largely due to difficulties created by focal localization of fringes produced by highly curved objects. Collimation is critical and it is necessary to image a point source precisely at the back focal plane of the objective in order to illuminate the object with parallel light at normal incidence and at the same time the object must be viewed critically in focus. With the small field of view, small working distance and small depth of focus, some delicate adjustment is involved.

(1) Some spherules (a minority) give very perfect regular Newton's ring interference fringe patterns, e.g. Figure 1e and Figure 1f $(\times 800)$. The remarkable perfection of circularity of these, which are micro-topographic contour fringes, is still maintained when the object is rolled to other regions of contact on the reference flat. This establishes that such objects are near perfect spheres. Clearly they must have been formed from small fluid glass droplets, subject only to surface tension forces, i.e. from droplets in free flight in a vacuum.

The notable smooth conformity of the fringes is a feature which shows that the specularity is today still very high, whatever the ages of the objects, which might even be many many millions of years, since ages between $3-5 \times 10^{9} \mathrm{yr}$ are reported for various Lunar rocks. Clearly objects of such smooth specularity could not have suffered any erosion or leeching effects either from atmosphere, or liquid nor indeed through any solid friction occasioned by rolling.

The fringe contrast permits assessment of a rough value of the refractive index. It is probably that for many of the objects the refractivity lies in the range 1.50-1.55.

(2) Several spherules (Figure $2 \mathrm{a}$ and $2 \mathrm{~b} \times 800$ ) show decisive evidence from the fringe pattern of having suffered collision damage, two being selected for illustration. Figure $2 \mathrm{a}$ reveals an impact crack, some half a light wave deep, whilst Figure $2 \mathrm{~b}$ shows clear evidence of cracking and chipping. It is reasonable to argue that these effects arose through impact on landing, thus adding further evidence that many spherules have been high-speed projectiles.

Not all spherules show smooth surface finish. Figure $2 \mathrm{c}$ shows small scale microwrinkles. Figure $2 \mathrm{~d}$ shows the region surrounding an impact crater.

(3) The glassy cylinders found, even if the long sides appear microscopically straight yet all show micro-waists when examined interferometrically. Figure $2 \mathrm{e}(\times 1000)$ suggests that this cylindrical object was probably a segment of a thin jet or filament of molten glass, a filament which originally broke up into both droplets and round-ended cylinders. Cylinders of fluid would, through surface tension, try to pull themselves 


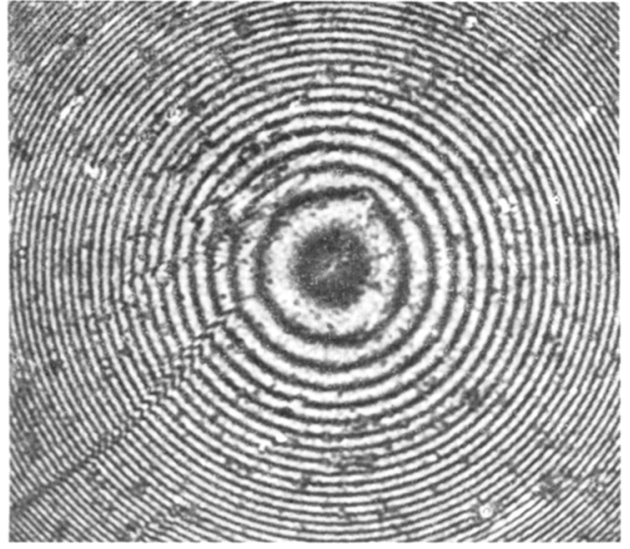

$2 a$.

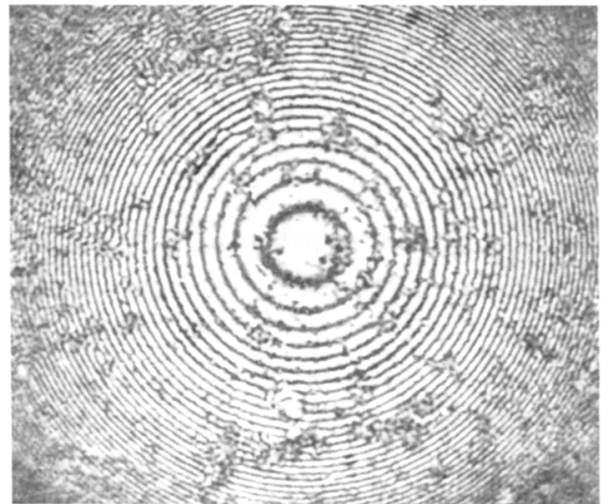

2c.

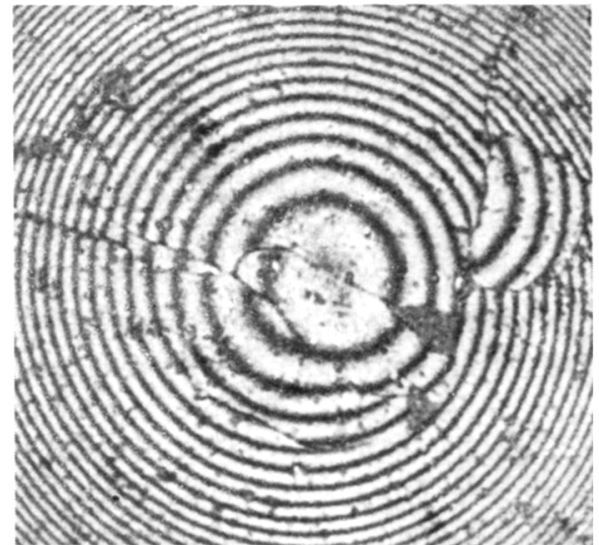

$2 b$.

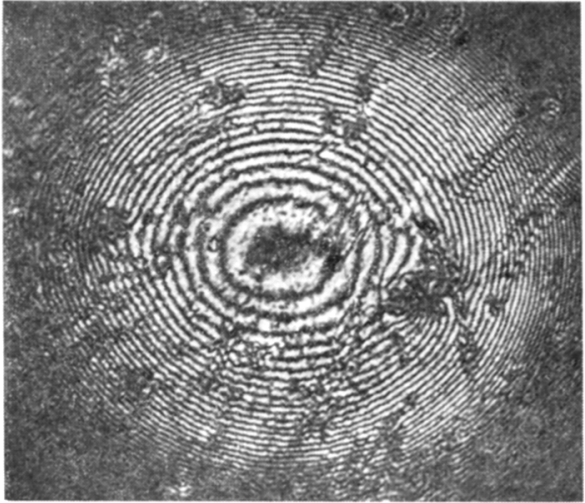

2d.

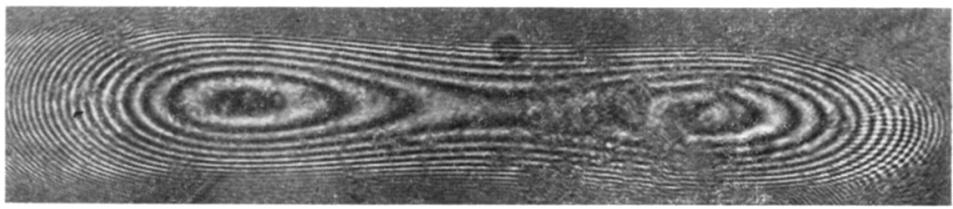

2 e.

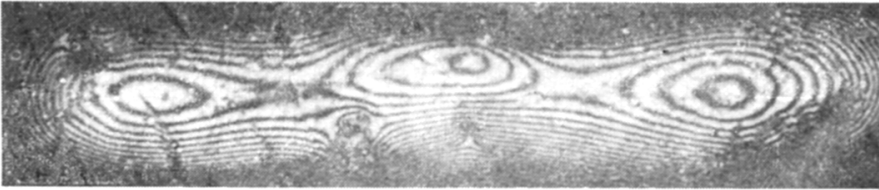

$2 f$.

Figs. 2a-f. 


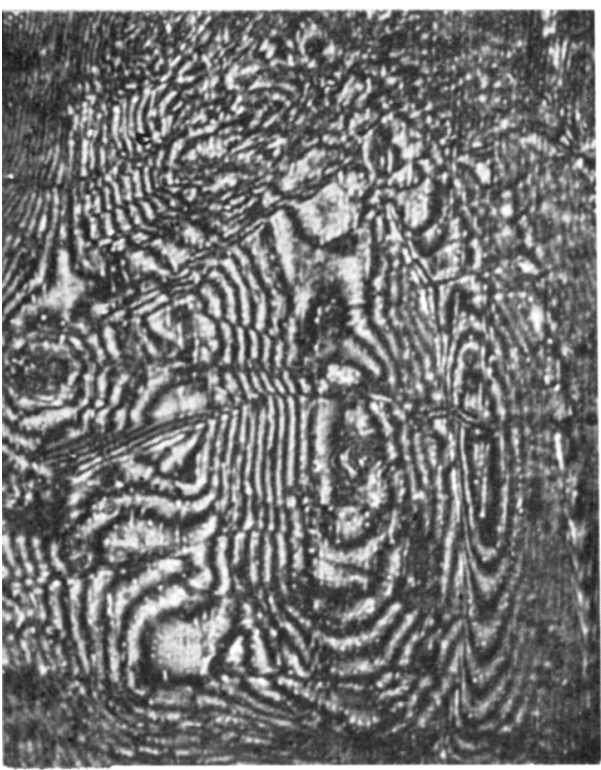

3a.

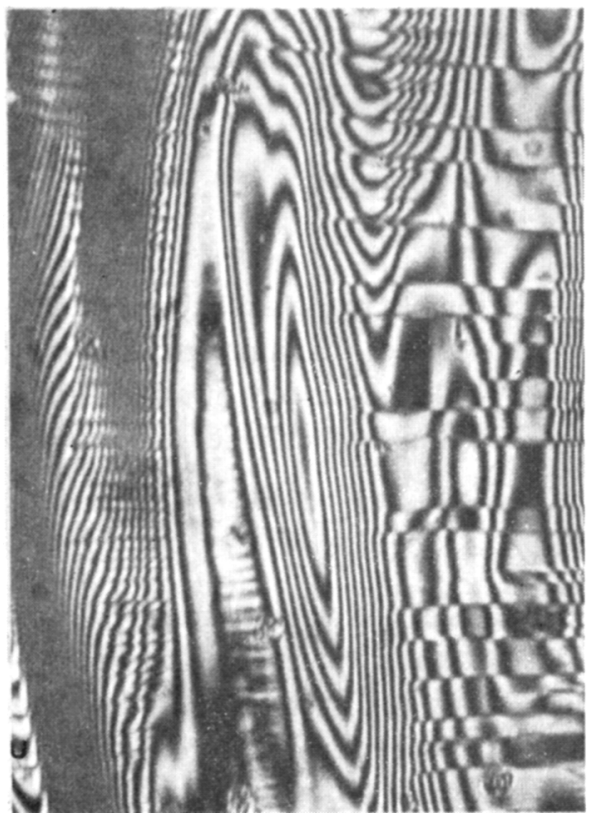

3c.

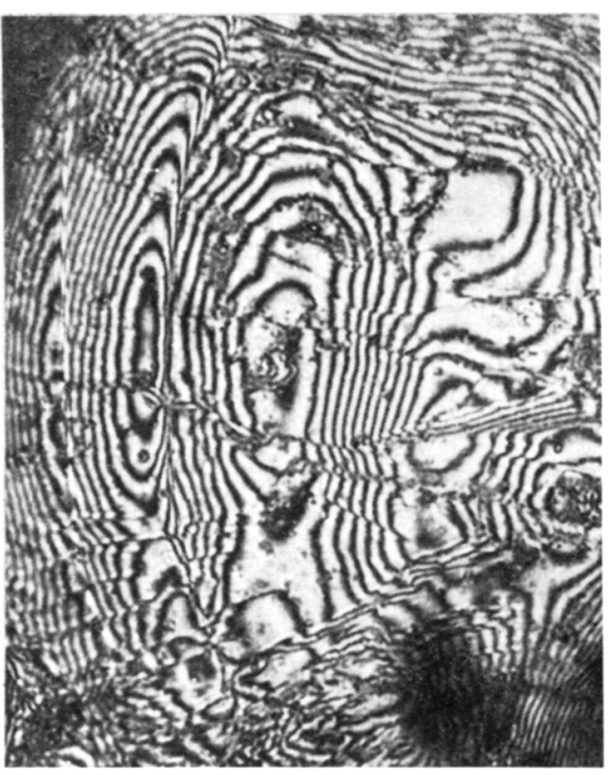

3b.

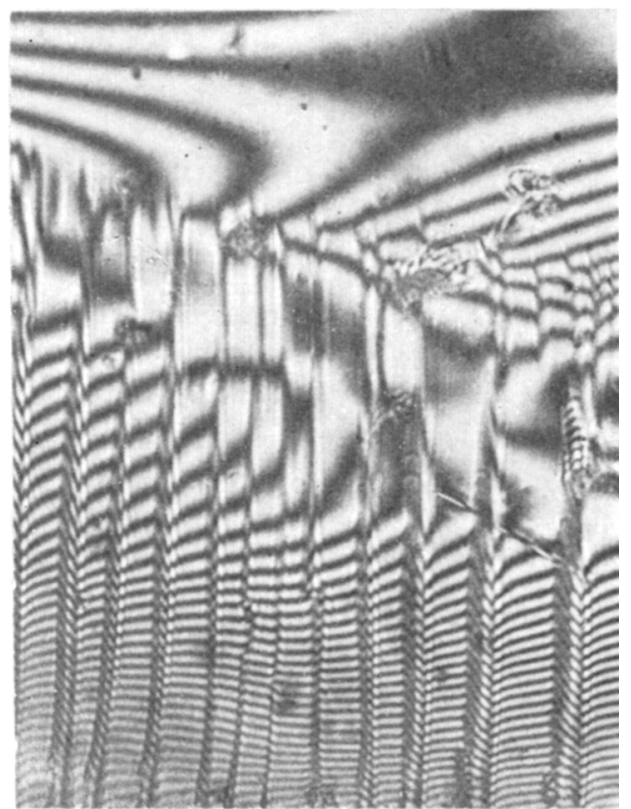

$3 d$.

Figs. $3 \mathrm{a}-\mathrm{d}$. 


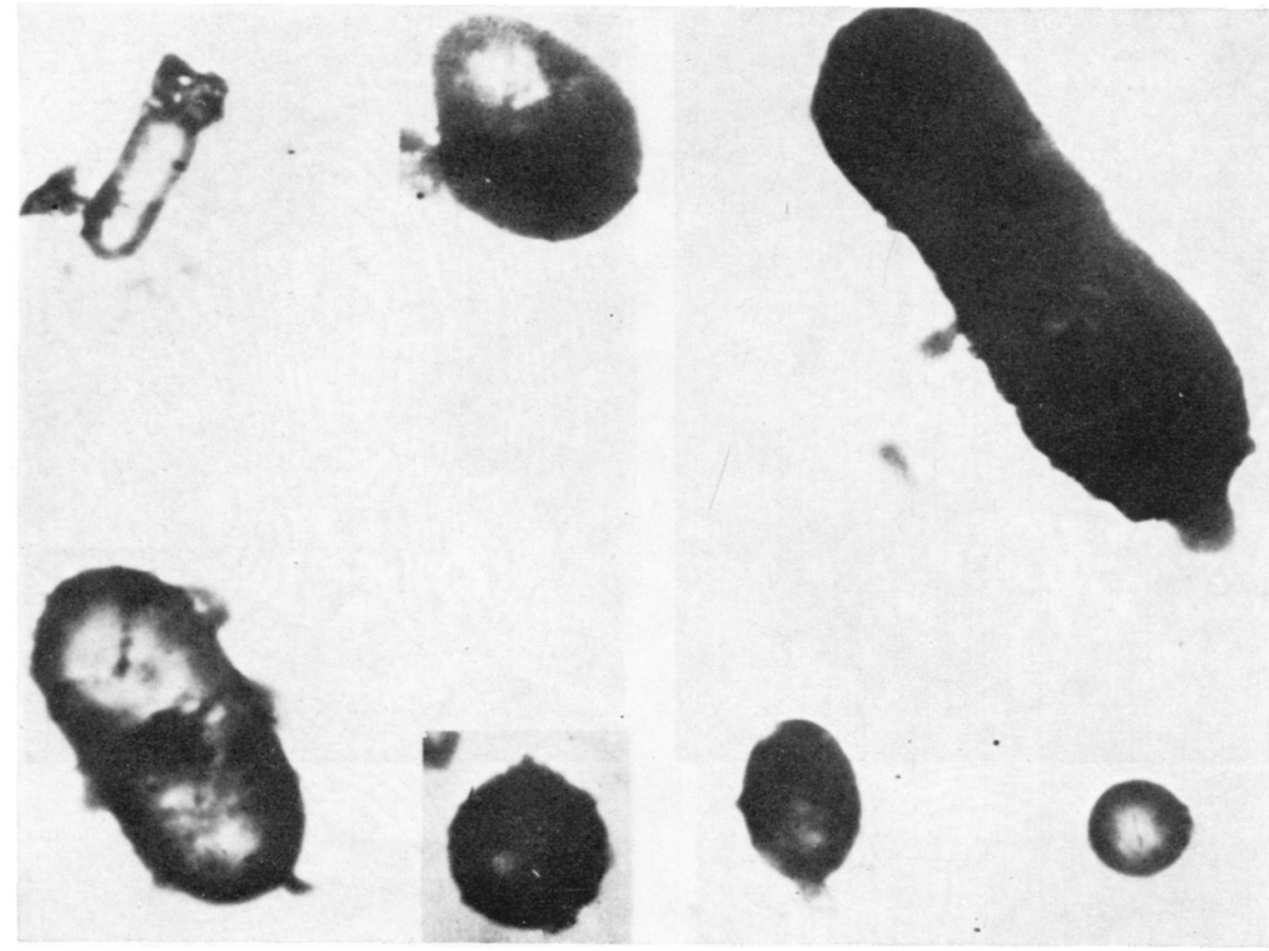

$3 e$.

Fig. 3e.

into droplets. Of course liquid glass near to solidification has high viscosity and clearly this object froze-in before splitting up. It will be shown later that amongst the micro objects there is a complete sequence of shapes from waisted cylinder, through dumbbell to parted droplets. Figure $2 \mathrm{f}$ is a case wherein a thin long cylinder has tried to split into three droplets, but froze-in.

\section{B. GLASS CHIPS}

The Lunar fines contain large number of fragmental pieces of glass, mostly brown in colour, giving the appearance of having been broken down by violent shock. Interferograms $(\times 500)$ for two typical such fragments are shown in Figures 3a, 3b. One can very closely simulate such discontinuous fringe patterns by crushing ordinary glass rod with hammer blows. For example numerous fragments of such crushed shocked glass give interferograms such as Figure $3 \mathrm{c}$ and $3 \mathrm{~d}$. The typical discontinuous fracture structure in these so resembles that in the Lunar glass fragments, that it is clear that some violent mechanical shock has operated equally on this Lunar material.

One notable Lunar chip, $1 \mathrm{~mm}$ in length, shown in Figure $4 \mathrm{a}$ gave the striking monochromatic interference fronge pattern of Figure $4 \mathrm{~b}$. By alternatively using whitelight it is established that the surface is spherically concave. Here is a remarkably smooth 


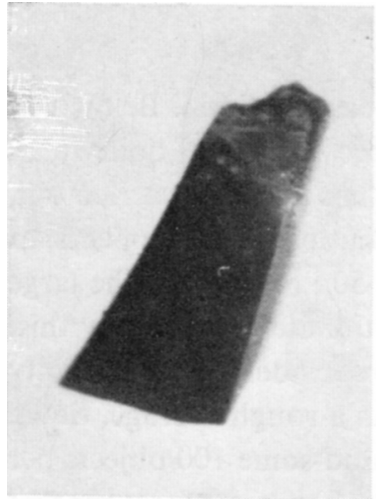

$4 a$.

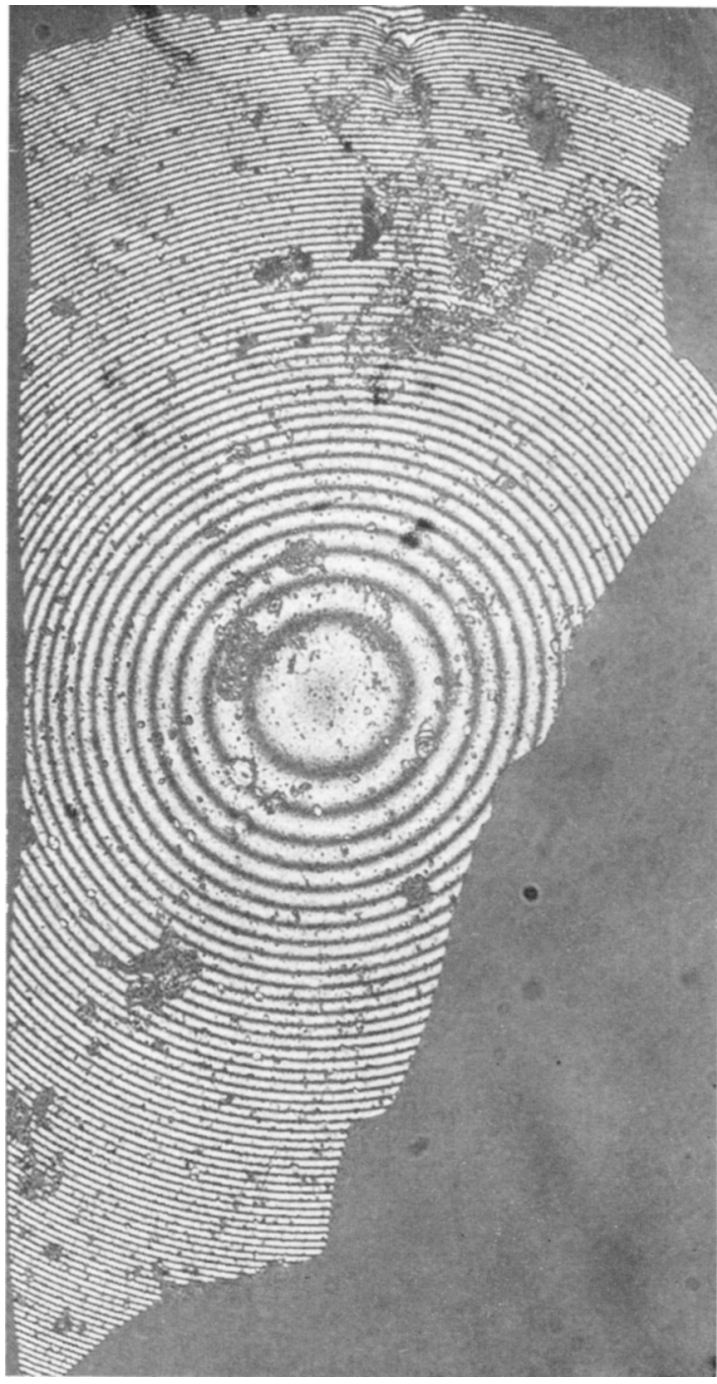

$4 b$.

Figs. $4 \mathrm{a}-\mathrm{c}$.

almost perfectly spherical hollow, of radius of curvature, obtained from the fringes as almost exactly $0.5 \mathrm{~cm}$. A reasonable conjectured explanation of this is shown by the diagram Figure $4 \mathrm{c}$. It is suggested that a larger piece of glass formed containing within itself a near spherical vacuole of $1 \mathrm{~cm}$ diam. This broke up and the chip, under discussion, as shown, shows a section of the highly specular $1 \mathrm{~cm}$ diam vacuole.

As an alternative, it is to be noted that some of the fragments of laboratory crushed glass do show roughly spherical small hillocks on occasion, but they are irregular and local small pips. Only one small depression was found in a considerable number of crushed particles. 


\section{MICRO OBJECTS}

It was possible to hand pick objects down to sizes of some $0.1 \mathrm{~mm}$ diam. Below this the glassy objects were only photographed. A surprisingly large number of quite small specular objects exist in the dust. Although a careful statistic has not been carried out, the impression is gained that there is a discontinuity in distribution. There appears, at first sight, to be a paucity of objects between the $100 \mu$ and $50 \mu$ range, and the large numbers appear between some $50 \mu$ and $10 \mu$, with proportionately less below this. Statistical sampling from a mere $5 \mathrm{gm}$ total must be subject to considerable uncertainty and little importance should be placed on these estimates. As a rough average, down to the resolution of a $4 \mathrm{~mm} 0.9 \mathrm{NA}$ lens, there have been found some 100 objects per approximate milligram smear of Lunar dust. The objects vary in character from spheres through to waisted cylinders and are best illustrated photographically as in Figure 3e. The size range covered is 0 to $20 \mu$ in this illustration. Though most are amber in colour there is a colour spread from deep blue to black, transmission from almost transparency to opacity and a range from smooth uniformity to those containing clearly defined inclusions or vacuoles. These are variously spheres, egg-shapes, pearshapes, dumb-bells and cylinders. One can track through the complete sequence expected from a fluid cylinder trying to break up into droplets and samples exist of a complete range of the fronzen-in states expected from such transitions. Some of the larger of these micro-objects have given reasonable interferograms.

When reviewing various mechanisms to account for the origin of these obviously pre-molten materials the possibility was considered that the objects could be in a sense artefacts. The question asked was whether the hot exhaust gases from the descending space-craft could have possibly melted some of the numerous small glass fragments in the dust and thus artificially created the spherules and other molten objects. A request to NASA was therefore put in asking for Apollo 12 material picked up as far as possible from the landing craft. This was granted and examination of this material completely excludes the idea that the rockets have anything at all to do with the objects. These objects are undoubtedly indigenous to the Moon prior to landing.

\section{Apollo 12}

Three 0.5 gm Apollo 12 samples were received, namely (a) contingency sample 12070.34 picked up some $10 \mathrm{~m}$ from the spacecraft landing site, (b) documented sample 12057.70 obtained from the bottom of the collecting box whose total material was collected over the range 80 and $330 \mathrm{~m}$ distant from the landing site, and (c) selected samples fines 12001.70 all picked up $120 \mathrm{~m}$ from the site.

The extractable objects from all three locations are very closely similar. Figure $5 \mathrm{a}$ and Figure $5 \mathrm{~b}$ are representative typical groups. There is close identity between Apollo 11 and Apollo 12 objects. Even blue as well as amber objects were extracted. For briefness only the above two representations are included here. Not only is there close identity in shapes, the interferograms are also closely related. 

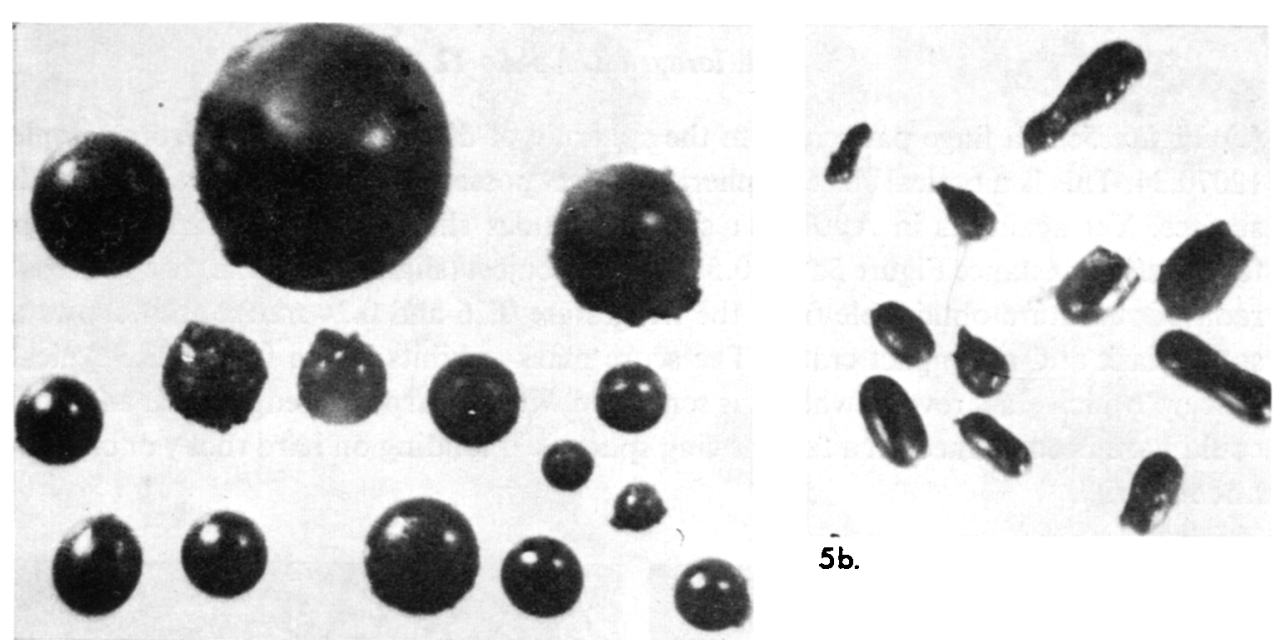

$5 b$.

5 a.
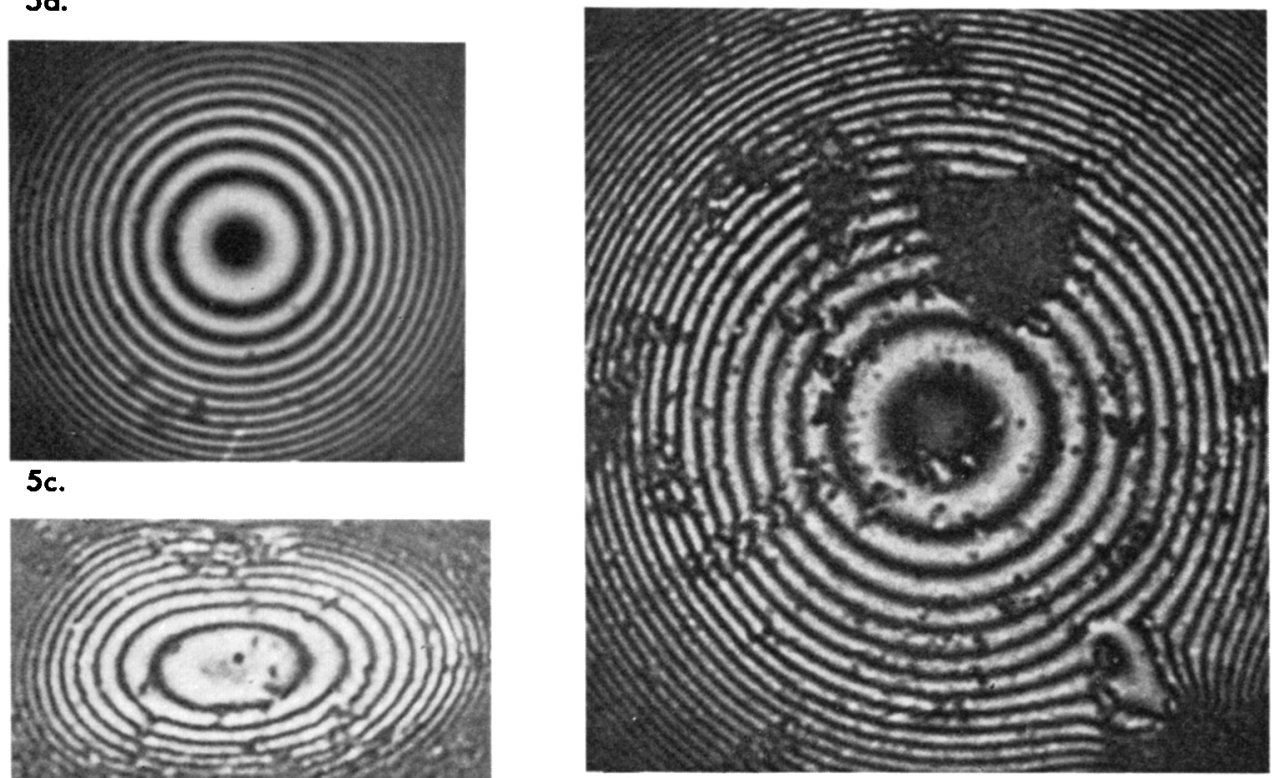

$5 c$.

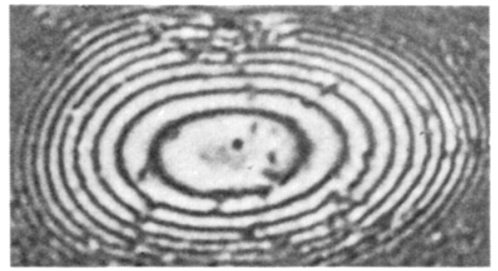

$5 e$.

$5 d$.

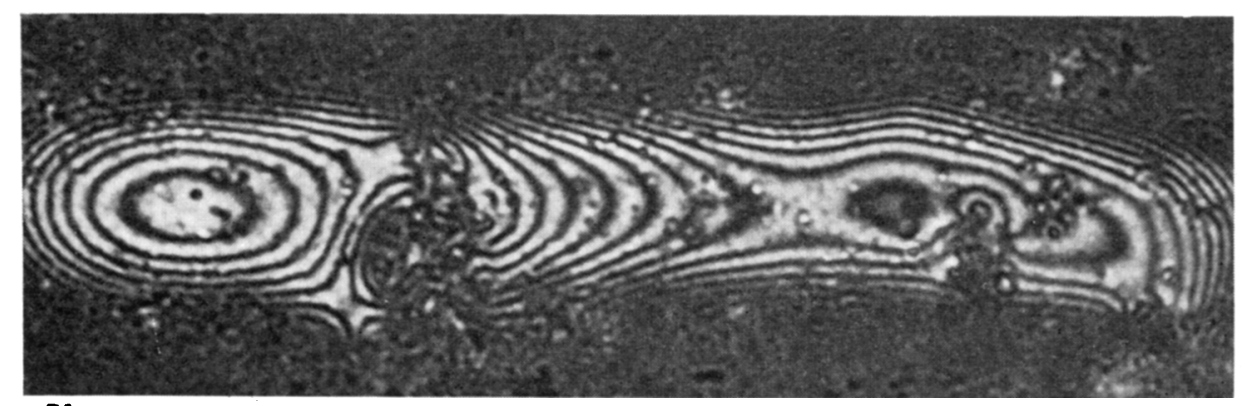

$5 f$.

Figs. 5a-f. 


\section{Interferogram. Apollo 12}

(1) Figure $5 \mathrm{c}$ is a finge pattern from the spherule of diameter $0.24 \mathrm{~mm}$ from sample 12070.34. This is a typical closely spherical object possessing a highly specular smooth surface. Yet again, as in Apollo 11 some spherules show surface cracks and other features. For instance Figure $5 \mathrm{~d}$ is a $0.5 \mathrm{~mm}$ diam object (slightly elliptical, for principal radii of curvature obtainable from the fringes are 0.26 and $0.24 \mathrm{~mm}$ ) and it shows a score mark and an impact crater. The score mark exhibits in the fringes the typical 'pile up' which glass reveals when it is scratched with a sharp hard edge. Such a furrow could have been formed on a fast moving spherule if landing on hard rocky or crystalline material.

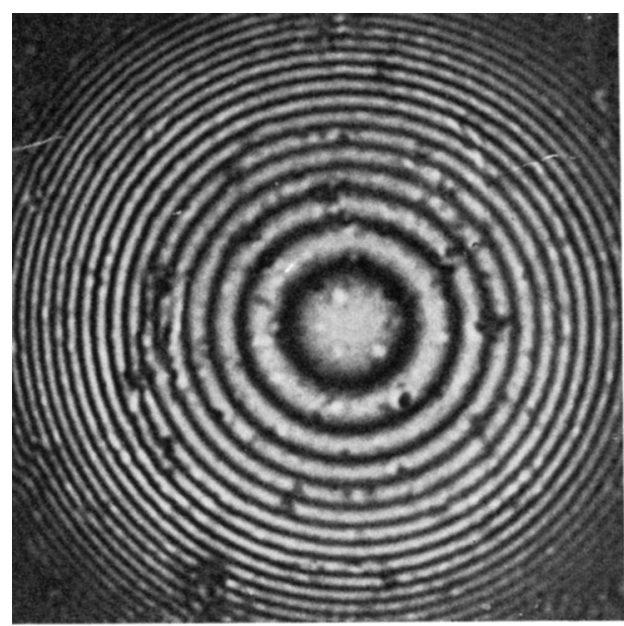

6a.

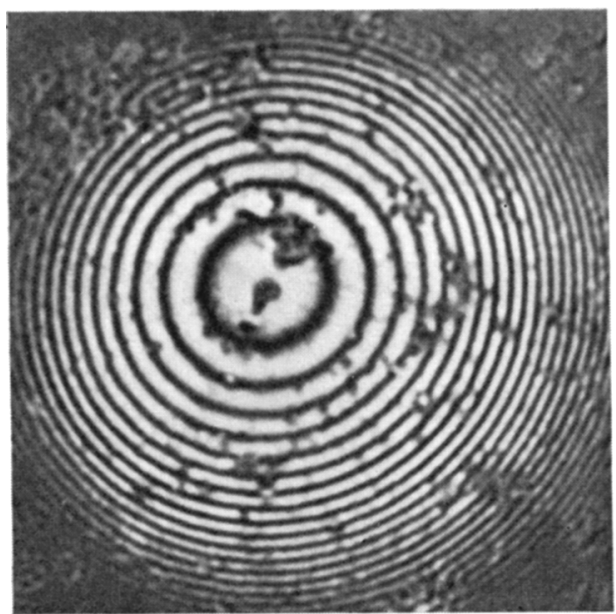

6c.

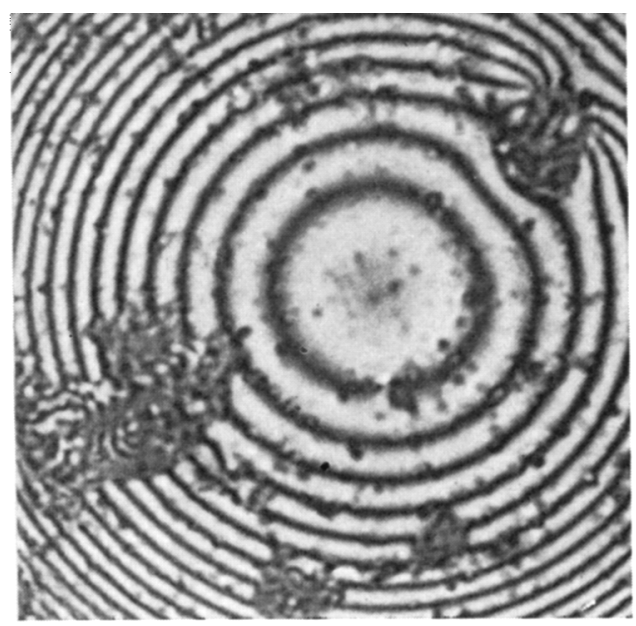

ob.

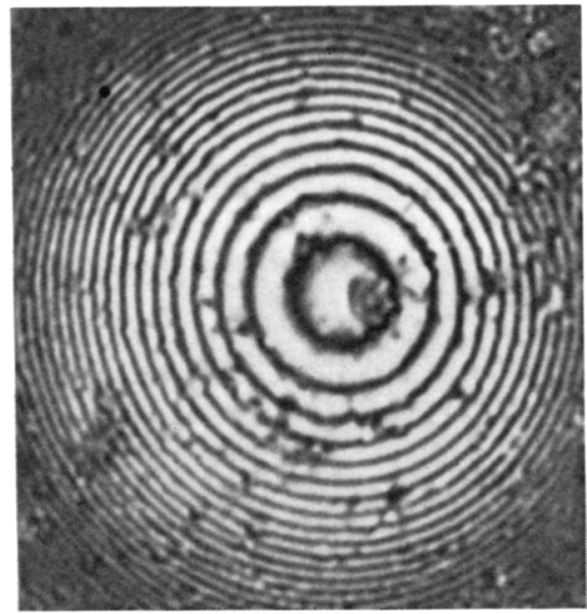

6d.

Figs. 6a-d. 


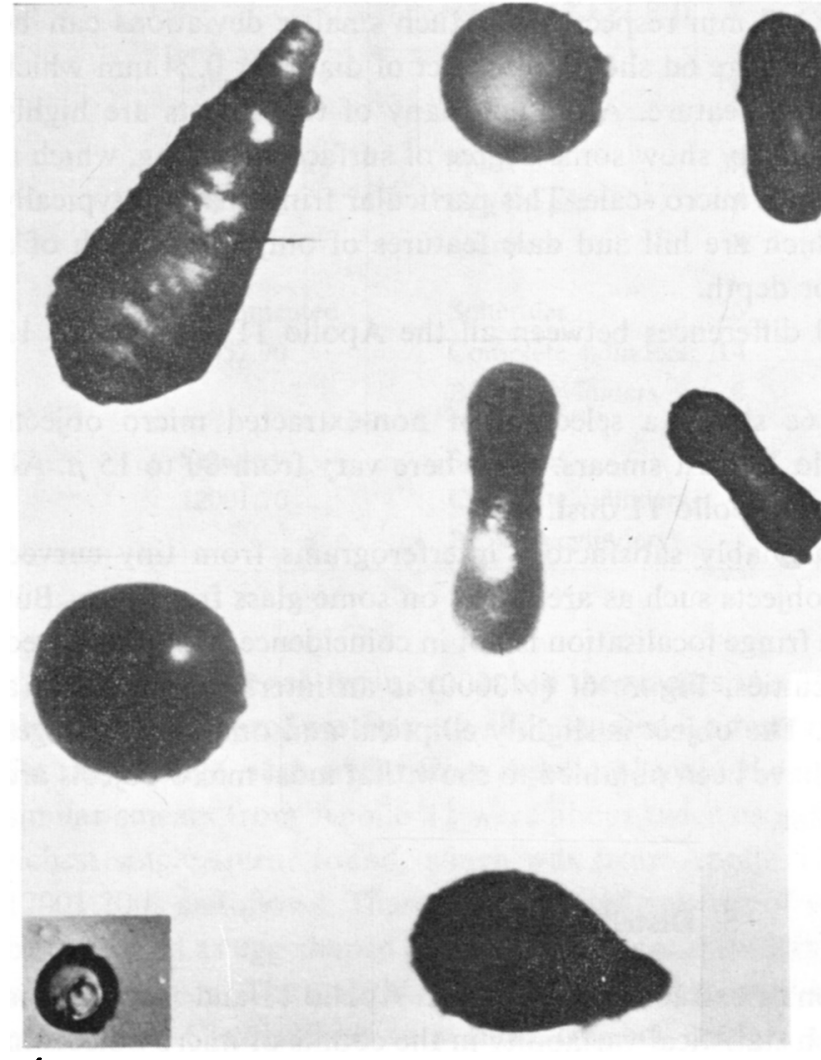

6e.
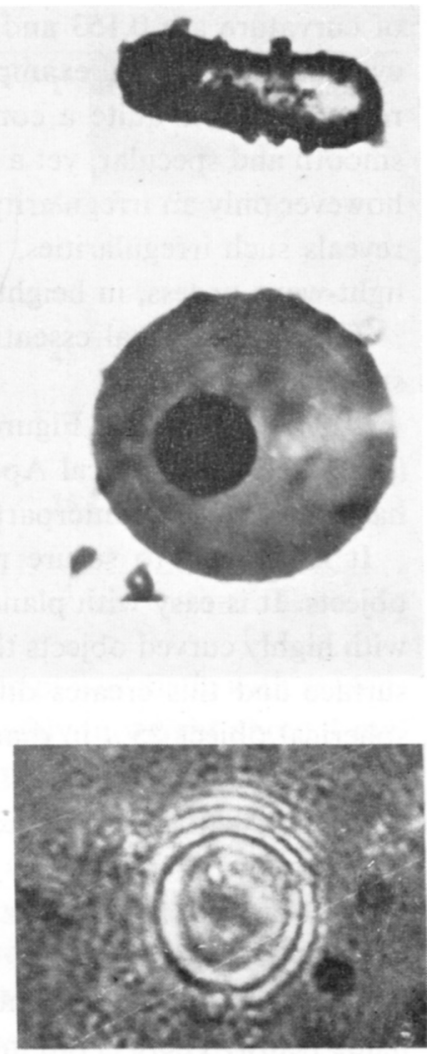

6f.

Figs. 6e-f.

Figure $5 \mathrm{e}$ is the interferogram from a characteristically egg-shaped object in which the principal radii are 0.18 and $0.05 \mathrm{~mm}$ respectively. Many other such object have been examined in detail. Figure $5 \mathrm{f}$ is the rough fringe pattern given by an elongated cylinder trying to pull itself into droplets but freezing in during the procedure, a feature often seen in Apollo 11 objects.

(2) Interferograms for objects from the second source (documented sample 12057.70 follow. Figure $6 \mathrm{a}$ is from a familiar high quality type of spherule. It has a diameter of $0.3 \mathrm{~mm}$. Figure $6 \mathrm{~b}$ is a striking pattern from a $0.6 \mathrm{~mm}$ spherule. So good is the fringe definition that there is no difficulty in easily seeing fringes well beyond the 50th order, indeed almost up to twice this. This is proof of a very smooth surface specularity. Some patches of adhering dust (it is very tenacious) still remain. A well defined microcrater of some two light waves in depth can be identified.

(3) The selected sample No. 12001.70 is a virtual repetition of what has already before. Thus Figure $6 \mathrm{c}$ is an example which illustrates how easily interferometry can show up even slight deviation from sphericity here a mere $4 \%$, for the principal radii 
of curvature are 0.153 and $0.147 \mathrm{~mm}$ respectively. Much smaller deviations can be evaluated. As a final example Figure 6d shows an object of diameter $0.24 \mathrm{~mm}$ which reveals what is quite a common feature. Although many of the objects are highly smooth and specular, yet a majority show some degree of surface wrinkling, which is however only an irregularity on a micro scale. This particular fringe pattern typically reveals such irregularities, which are hill and dale features of only a twentieth of a light-wave or less, in height or depth.

There are no real essential differences between all the Apollo 11 and Apollo 12 specimens.

(4) Micro objects. Figure 6e shows a selection of non-extracted micro objects ( $\times 1000)$ from a typical Apollo 12 dust smears. Sizes here vary from 80 to $15 \mu$. All have their close counterparts in Apollo 11 dust.

It is difficult to secure reasonably satisfactory interferograms from tiny curved objects. It is easy with plane objects such as are found on some glass fragments. But with highly curved objects the fringe localisation is not in coincidence with the curved surface and this creates difficulties. Figure $6 \mathrm{f}(\times 3000)$ is an interferogram from a spherical object $25 \mu$ in diam. The object is slightly elliptical and only a few fringes are secured. Yet enough data have been obtained to show that most micro objects are reasonably smooth and specular.

\section{Distribution}

Comparison of the distribution of extracted objects from Apollo 11 and Apollo 12 is made below. There is too much statistical variability in the counts of micro objects in the separate smears to make differences of such objects very significant.

In Apollo 11 the objects total 207 distributed thus (sample 10084.15) (see Table I).

From this some $86 \%$ are largely spherical and 14\% cylinders. The three Apollo 12 samples are so small $(0.5 \mathrm{gm}$ each $)$ that it is wiser to group objects together as indicated in Table II.

Pro rata the Apollo 12 samples, together, are about twice as rich in objects as the Apollo 11 sample.

TABLE I

\begin{tabular}{lc}
\hline Object & Number \\
\hline & \\
Very clear spherules & 6 \\
Red-brown spherules & 32 \\
Small spherules & 56 \\
Rough spherules & 41 \\
Broken spherules & 6 \\
Spherules with tails & 12 \\
Grey spherules & 25 \\
Cylinders & 29
\end{tabular}


TABLE II

\begin{tabular}{|c|c|c|c|c|}
\hline Sample & Object & Number & Total & $\%$ cylinders \\
\hline Contingency & Spherules & 39 & & \\
\hline \multirow[t]{2}{*}{12070.34} & Egg shapes & 3 & 50 & $16 \%$ \\
\hline & cylinders & 8 & & \\
\hline Documented & Spherules & 25 & & \\
\hline \multirow[t]{2}{*}{12057.70} & Complete cylinders & 14 & 45 & $45 \%$ \\
\hline & Broken cylinders & 6 & & \\
\hline Selected & Spherules & 24 & & \\
\hline \multirow[t]{2}{*}{12001.70} & Complete cylinders & 7 & 35 & $32 \%$ \\
\hline & Broken) cylinders & 4 & & \\
\hline
\end{tabular}

There is a wide scatter in content in the smears. An attempt was made with reasonable success to produce on each slide studied a smear roughly in weight about $1 \mathrm{mg}$. On the average, each smear from Apollo 11 yielded something like 100 objects whilst similar smears from Apollo 12 were about twice as rich. The detailed content of the richest single smear found, which was from Apollo 12, in the selected sample No. 12001.70 is as follows. There are 230 small spheres of very good shape, 55 which can be described as egg-shaped and 30 which were cylinders, some with and some without obvious waists. Thus of this total of 315 objects only some $9 \frac{1}{2} \%$ can be described as cylindrical. Clearly with very small objects, surface tension forces tend to favour sphericity. It is notable that this represents $3.15 \times 10^{9}$ objects per $\mathrm{kg}$ within range of optical resolution. An electron microscope study might yield numbers of still smaller objects.

\section{Discussion}

There seems to be a real difference between the extractable ('macro') objects and the much more abundant 'micro' objects. It is proposed that several distinct mechanisms might be involved. It can in the first instance be considered that the 'macro' objects are created through a meteoric impact which raises the temperature both of itself and of the rock struck to that of melting point. Consequently there would be violent splashing in possibly three distinct ways. Firstly droplets would be flung out violently and the smaller would freeze rapidly into hard glassy spherules. These would impact on landing producing the features already described. Fine threads or jets of glass could also be thrown out and these would break up into the observed cylinders. The larger blobs of glass would not have time to freeze and onlanding would both form flat 'pancakes' (as reported) and also possibly these would produce secondary splashing with more spherules etc. created.

It might be expected that this same impact which leads to drop formation could also pulverise surrounding areas and thus hurl up a cloud of small solid particles. It would 
be the passage of the frozen spherules through such a cloud which would explain the micro-craters seen on so many spherules.

This proposed mechanism can account both for the widespread distribution of glass lumps and glass spherules. It certainly does not explain the very high content of tiny fragmented pieces of glass in the Lunar soil and neither does it explain the enormous numbers of micro objects, nor the fact that many of them are broken. So a subordinate hypothesis is proposed. It is suggested that repeated multiple impacts (and clearly enormous numbers of impacts have happened) lead each to the passage of a violent shock wave across the surface of the Moon, sufficiently violent to break down existing glass to the vast number of small fragments observed. This explains too the shocked nature of so many glass fragments. Then, on occasion, it is conjectured that a more than usually violent impact happens and as a consequence the resulting shock front which is propagated has now a high temperature, sufficient to melt locally some of the small glass fragments. This then creates the micro objects which are predominantly of the very same colour and character as the neighboring shattered small glass fragments so abundant in the soil.

It is not surprising then if later impacts producing later shock waves can break up some micro objects, as is found. It is very difficult to account otherwise for broken micro spherules and broken micro cylinders. For even if these had been projectiles their momenta could hardly lead to shattering on impacting into the soft dust.

The smooth interferometric specularity observed often must be associated with the formation of the objects in the good vacuum conditions on the Moon. For an interferometric examination carried out on the tiny glass 'ballotini' made industrially for filters etc. by hot flames blasting onto glass fibres, in the Earth's atmosphere, shows fringe patterns which give evidence of crude rough surface wrinkling which bear no resemblance to Lunar spherule interferograms at all. Nor do Earth tektites show surface features resembling Lunar glassy objects.

A possible alternative mechanism for production of the glassy Lunar objects can be conjectured as follows. Let it be proposed that a really large violent meteoric impact melts a big region of rock, creating in effect a veritable extensive lake of very hot molten glass. Now let this be followed by a violent eruption of gas from below. This eruption will be explosively violent, into the effective high vacuum of the Moon's 'atmosphere'. Such a gas blow might be prolonged and could produce a veritable fountain of an enormous number of tiny glass droplets, hurled far and wide. Even the micro objects might also be envisaged as a consequence of sufficiently violent gas eruptions through a molten lake of glass.

However one still has to explain the existence of the fracture-reduced fragments of glass in the soil. Maybe the two mechanisms proposed could both operate.

If this hot lake mechanism is valid, one might expect that if future astronauts land inside a crater then they could find that its inner floor consisted of solidified glass, of the same constitution as the numerous typical spherules.

Finally it is notable that although two sites of the Apollo missions are some 800 miles apart, there is such close identity in the nature of the spherules. In addition to 
this is the fact that the retrieved 100 gm sample brought back by the Russian automatic retrieval rocket, although picked up from a site 1500 miles away from one of the Apollo sites, also shows at least one glass spherule.

There seems every indication that the whole Moon is probably covered with these objects and the numbers of them must be very vast. 